\title{
Limiting fragmentation in high-energy nuclear collisions at the CERN Large Hadron Collider
}

\author{
Pragati Sahoo, Pooja Pareek, Swatantra Kumar Tiwari, and Raghunath Sahoo* \\ Discipline of Physics, School of Basic Sciences, Indian Institute of Technology Indore, Simrol, Khandwa Road, Indore, 453552, India
}

(Received 9 June 2018; revised manuscript received 12 February 2019; published 9 April 2019)

\begin{abstract}
The hypothesis of limiting fragmentation (LF), or, as it has been otherwise recently called, longitudinal scaling, is an interesting phenomena in the high-energy multiparticle production process. This paper discusses different regions of phase space and their importance in hadron production, giving special emphasis on the fragmentation region. Although it was conjectured as a universal phenomenon in high-energy physics, with the advent of higher center-of-mass energies, it has become prudent to analyze and understand the validity of such a hypothesis in view of the increasing inelastic nucleon-nucleon cross section $\left(\sigma_{\text {in }}\right)$. In this work, we revisit the phenomenon of limiting fragmentation for nucleus-nucleus $(A+A)$ collisions in the pseudorapidity distribution of charged particles at various energies. We use energy-dependent $\sigma_{\text {in }}$ to transform the chargedparticle pseudorapidity distributions $\left(d N_{\mathrm{ch}}^{A A} / d \eta\right)$ into differential cross section per unit pseudorapidity $\left(d \sigma^{A A} / d \eta\right)$ of charged particles and study the phenomenon of LF. We find that in $d \sigma^{A A} / d \eta \mathrm{LF}$ seems to be violated at Large Hadron Collider (LHC) energies while considering the energy-dependent $\sigma_{\text {in }}$. We also perform a similar study using the A Multi-Phase Transport model with a string melting scenario and also find that LF is violated at LHC energies.
\end{abstract}

DOI: 10.1103/PhysRevC.99.044906

\section{INTRODUCTION}

Understanding the particle production in high-energy nuclear collisions is always fascinating. Particle production in high-energy collisions happens from three different regions: the projectile, the target, and the central region. Particles emitted from the outer region are called projectile/target fragments. There are various nuclear fragmentation mechanisms discussed in the literature [1,2]. The most important are a sudden fragmentation by explosive mechanisms, such as shock waves [1], and a slow fragmentation by the "fission" of the spectator regions, mainly because of the interactions with the particles or fragments emitted from the participant region at transverse angles in the center-of-momentum system [1]. The latter is a purely low-energy nuclear phenomenon, whereas the former is more applicable to relativistic domain of energies. During the late 1960s, the hypothesis of limiting fragmentation became important to understand particle production [2,3]. According to this hypothesis, the produced particles, in the rest frame of one of the projectiles, become independent of center-of-mass energies, thus following a possible scaling (as a function of $\eta^{\prime}=\eta \pm y_{\text {beam }}$ ), known as limiting fragmentation (LF). As (pseudo)rapidity is a longitudinal variable, it is also called longitudinal scaling. Here $y_{\text {beam }}=$

*Corresponding author: Raghunath.Sahoo@cern.ch

Published by the American Physical Society under the terms of the Creative Commons Attribution 4.0 International license. Further distribution of this work must maintain attribution to the author $(\mathrm{s})$ and the published article's title, journal citation, and DOI. Funded by $S C O A P^{3}$. $\ln \left(\sqrt{s_{N N}} / m_{p}\right)$ is beam rapidity and $m_{p}$ is the mass of proton. There have been several attempts to understand the nature of hadronic interactions which lead to limiting fragmentation and the deviations from it [4-6].

It is expected that a central plateau develops at higher energies, which clearly separates the central rapidity from the fragmentation region. However, as such, there is no separating boundary between the central rapidity and the fragmentation region. The width of the fragmentation region is around two units in rapidity [7]. The fragmentation region thus is expected to be well separated from the central region only in very high energies, as the kinematically available rapidity region is much wider than four units in rapidity. Particle production in the fragmentation region is attributable to the valence quarks participating in hadronization, whereas in the central rapidity region, it is dominated by the midrapidity gluonic sources at high energies $[8,9]$. The central rapidity region is called the pionization region [7] and is shown in Fig. 1.

There have been several experimental efforts to understand particle production in both mid- and forward rapidities [10-17]. As LF is the focus of this paper, we discuss particle production in the forward rapidity region. The experimental observation of LF was first reported by the PHOBOS experiment at the Relativistic Heavy Ion Collider (RHIC) with charged particles [17]; later the STAR experiment also confirmed the hypothesis with inclusive photons in the forward rapidity [13]. The limiting fragmentation was observed by the UA5 experiment at CERN for $p p$ and $p \bar{p}$ collisions from 53 to $900 \mathrm{GeV}$ [18]. However, the ALICE experiment at the Large Hadron Collider (LHC) has reported a violation of the LF hypothesis for inclusive photons in $p p$ collisions with limited forward-rapidity coverage [19]. 


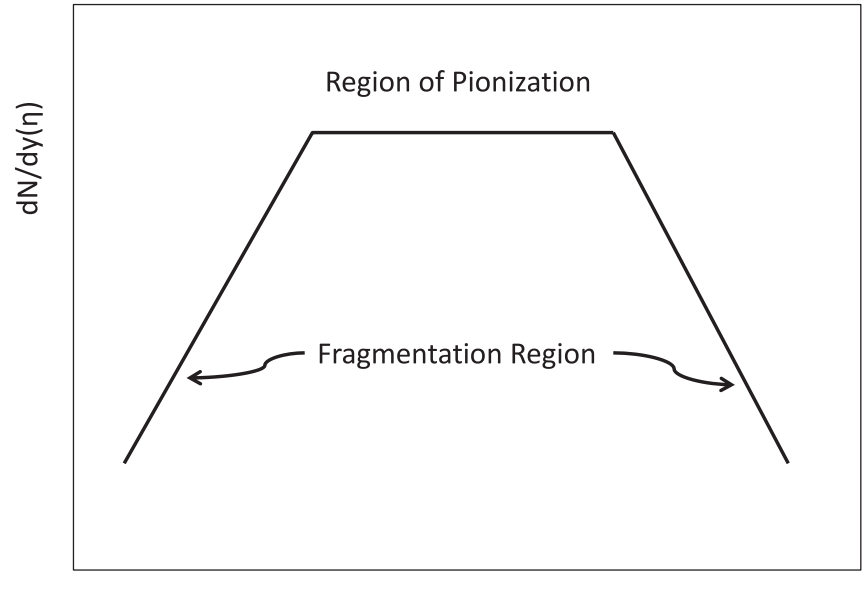

$-y(\eta)$

$y(\eta)$

FIG. 1. A schematic of (pseudo)rapidity distribution showing the pionization and fragmentation regions.

Various theoretical works [5,6,20-25] have reported the observation of limiting fragmentation phenomenon in heavyion collisions. Recently, limiting fragmentation in the era of RHIC and LHC has revealed a new concept, called the hypothesis of "energy-balanced limiting fragmentation" [26,27]. In Ref. [5], it is claimed that the cross section plays an important role in fragmentation regions. Marian [6] has shown that the LF phenomenon is observed in the differential cross section per unit pseudorapidity in proton+nucleus collisions at RHIC energies.

Our main aim in this work is to study the phenomena of LF for $A+A$ collisions in view of increasing inelastic particle production cross section from RHIC to LHC energies. The hypothesis of limiting fragmentation can be tested for both observables, namely the particle multiplicity density and the differential cross section. As LF is least explored in the case of differential cross section, this work focuses on the latter observable with a detailed discussion on multiplicity as well for a clear comparison of the expected results at the LHC energies. The total hadronic cross section does not remain constant from lower RHIC energies to the highest LHC energy but is a slowly increasing function of $\sqrt{s}$ [28]. Particle production in heavy-ion collisions depends on the hadronic cross section. Thus, a detailed study of the longitudinal scaling behavior in terms of cross section could be a prudent attempt. The longitudinal variables are expected to be sensitive to the available energy and the multiplicity of the produced secondaries. In this context, the study of possible longitudinal scaling of the final-state multiplicity as a function of collision energy becomes judicious, in view of increasing inelastic particle production cross sections at LHC energies. The paper is organized as follows: In Sec. II, we recapitulate the basics of Landau hydrodynamics and its connection with the limiting fragmentation hypothesis. In Sec. III, we present the methodology to calculate the differential cross section per unit pseudorapidity and discuss the results obtained using experimental data and A Multi-Phase Transport (AMPT). Finally, we summarize our findings in Sec. IV.

\section{LANDAU HYDRODYNAMICS AND LIMITING FRAGMENTATION HYPOTHESIS}

The angular distribution of the particles produced in highenergy collisions is described by the famous Landau model with relativistic hydrodynamics given by the conservation of energy momentum tensor, $\partial_{\mu} T^{\mu \nu}=0$ with a blackbody equation of state, $p=\epsilon / 3$, where $p$ is the pressure and $\epsilon$ is the energy density $[29,30]$. The Landau hydrodynamical model assumes complete thermalization of the total energy in the Lorentz contracted volume of the fireball, which makes the initial energy density to grow with collision energy [31]. The formulation given in Ref. [31] gives rise to the initial entropy of the system, which is produced in the thermalization process of the quanta of the system to follow a Gaussian distribution in the rapidity space. The width of the rapidity distribution is determined by the Lorentz contraction factor and is related to the speed of sound [32]. The multiplicity distribution in the rapidity space thus becomes $[29,30,33]$

$$
\frac{d N}{d y}=\frac{K s^{1 / 4}}{\sqrt{2 \pi L}} \exp \left(-\frac{y^{2}}{2 L}\right),
$$

where $L=\sigma_{y}^{2}=(1 / 2) \ln \left(s / m_{p}^{2}\right)=\ln (\gamma)$. Equation (1) can be rewritten as

$$
\frac{d N}{d y}=\frac{K s^{1 / 4}}{\sqrt{2 \pi y_{\text {beam }}}} \exp \left(-\frac{y^{2}}{2 y_{\text {beam }}}\right) .
$$

The conclusion from Ref. [31] shows that the hypothesis of limiting fragmentation comes naturally in Landau's model of multiparticle production. Following the LF hypothesis, when the rapidity distribution is seen from one of the projectiles' rest frame, i.e., by transforming to $y^{\prime}=y-y_{\text {beam }}$, the above expression for rapidity distribution becomes $(d N / d y=$ $\left.d N / d y^{\prime}\right)[31]$

$$
\begin{aligned}
\frac{d N}{d y^{\prime}} & =\frac{K s^{1 / 4}}{\sqrt{2 \pi y_{\text {beam }}}} \exp \left[-\frac{\left(y^{\prime}+y_{\text {beam }}\right)^{2}}{2 y_{\text {beam }}}\right], \\
& =\frac{K s^{1 / 4}}{\sqrt{2 \pi y_{\text {beam }}}} \exp -\left(\frac{y^{\prime 2}}{2 y_{\text {beam }}}+y^{\prime}\right) \exp \left(\frac{-y_{\text {beam }}}{2}\right), \\
& =\frac{1}{\sqrt{y_{\text {beam }}}} \exp \left(-\frac{y^{\prime 2}}{2 y_{\text {beam }}}-y^{\prime}\right) .
\end{aligned}
$$

For $y^{\prime}=0$, the distribution only depends on the Lorentz contraction factor, which is a function of collision energy. When we make the transformation, $y^{\prime}=y-y_{\text {beam }}$, the fragmentation region shifts by a factor $y_{\text {beam }}$, a value which increases with the collision energies, making the regions overlap with each other.

\section{LIMITING FRAGMENTATION AT THE LHC}

In this section, we study the limiting fragmentation phenomenon in the pseudorapidity distributions of differential cross sections of charged particles $(d \sigma / d \eta)$ for $A+A$ collisions at various center-of-mass energies starting from $19.6 \mathrm{GeV}$ to $5.02 \mathrm{TeV}$. Due to the lack of experimental data for $d \sigma^{A A} / d \eta$, we take the experimentally measured $d N_{\mathrm{ch}}^{A A} / d \eta$ at various collision energies. We transform $d N_{\mathrm{ch}}^{A A} / d \eta$ into $d \sigma^{A A} / d \eta$ using nucleon-nucleon inelastic cross sections $\left(\sigma_{\text {in }}\right)$ 


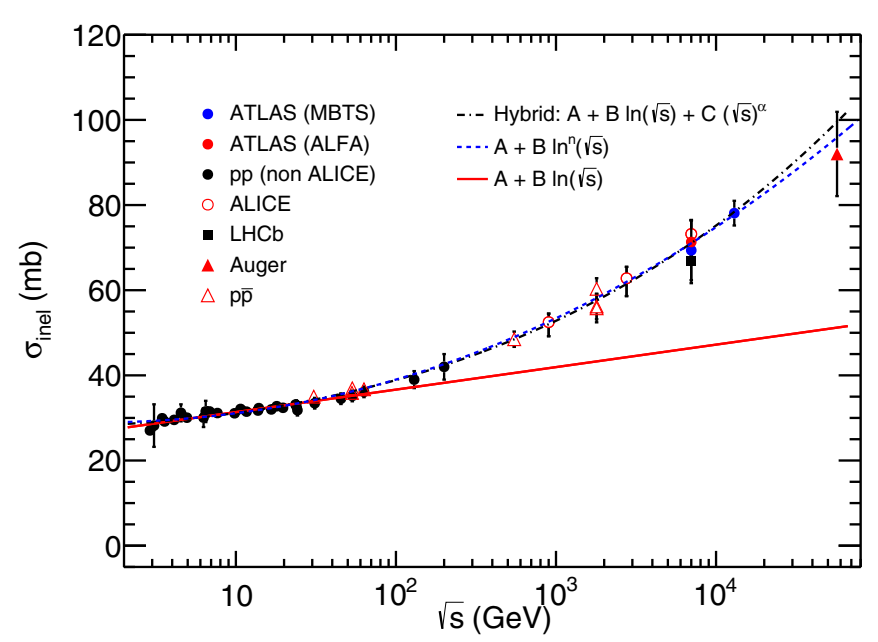

FIG. 2. The inelastic cross section as a function of $\sqrt{s}$. The symbols are experimental data [34-37] and the fitted lines are phenomenologically motivated functions.

for different energies applying the method discussed below. A very detailed study is needed to make the connection possible. Recent studies [6] show that the longitudinal scaling of the differential cross section per unit pseudorapidity is observed in the experimental data for higher RHIC energies. The rationale behind our work is to bring in the direct center-of-mass energy dependence of $\sigma_{\text {in }}$, which has a different low-energy behavior up to the top RHIC energy in comparison to the LHC energies. This is also observed from the experimentally measured values of $\sigma_{\text {in }}$ [34-37], which are shown in Fig. 2. In this figure, we show the variation of $\sigma_{\text {in }}$ with collision energy. It is clearly seen that there is a very slow rise of $\sigma_{\text {in }}$ at lower collision energies up to the top RHIC energy. We have fitted the experimental data with various phenomenologically motivated functions to understand the energy-dependent behavior of $\sigma_{\text {in }}$. A logarithmic function, $A+B \ln (\sqrt{s})$, with $A$ and $B$ as free fitting parameters, explains the data only up to RHIC energies. This function seems to deviate completely after the top RHIC energy. The $\sigma_{\text {in }}$ beyond the top RHIC energy do not follow a logarithmic behavior. To study the complete energy-dependent behavior, we have used a hybrid function, $A+B \ln (\sqrt{s})+C(\sqrt{s})^{\alpha}$, which combines logarithmic and a power law to fit the data. Here $A, B, C$, and $\alpha$ are free parameters. This hybrid function explains the data from lower to higher energies. We have also fitted the data with a function $A+B \ln ^{n}(\sqrt{s})$, where $A$ and $B$ are free parameters. A more detailed discussion could be found in Ref. [28]. This function seems to describe the data very well. These findings suggest that the logarithmic function alone cannot explain the data for higher energies, while the power of logarithmic function and the hybrid function mentioned above could explain experimental data from lower to higher energies shown in the figure. The $\sigma_{\text {in }}$ at LHC energies showing a different functional behavior than the lower energies necessitates a review of the hypothesis of limiting fragmentation.

Considering the crude approximation to the physical situation in the framework of the Landau hydrodynamical model of particle production, the relationship between the differential cross sections per unit pseudorapidity $\left(d \sigma^{p p} / d \eta\right)$ and the pseudorapidity distribution $\left(d N_{\mathrm{ch}}^{p p} / d \eta\right)$ of charged particles for $p p$ collisions is given as [38]

$$
\frac{d \sigma^{p p}}{d \eta}=\sigma_{\text {in }}\left(\frac{d N_{\mathrm{ch}}^{p p}}{d \eta}\right) \text {. }
$$

Now the relation of charged-particle pseudorapidity distribution in $A+A$ collisions with the charged-particle pseudorapidity distribution in $p p$ collisions using a two-component model, where the contributions from soft and hard processes in particle production are taken separately, is given as $[39,40]$

$$
\frac{d N_{\mathrm{ch}}^{A A}}{d \eta}=\frac{d N_{\mathrm{ch}}^{p p}}{d \eta}\left[(1-x) \frac{\left\langle N_{\mathrm{part}}\right\rangle}{2}+x\left\langle N_{\mathrm{coll}}\right\rangle\right] .
$$

Here $x$ and $(1-x)$ are the fractions of contribution to particle production from hard and soft processes, respectively.

Using Eq. (5) in Eq. (4), we get a relation between the differential cross section per unit pseudorapidity in $p p$ collisions and the charged-particle pseudorapidity distribution in heavy-ion collisions as follows:

$$
\frac{d \sigma^{p p}}{d \eta}=\frac{\sigma_{\mathrm{in}}\left(\frac{d N_{\mathrm{ch}}^{A A}}{d \eta}\right)}{\left[(1-x) \frac{\left\langle N_{\mathrm{part}}\right\rangle}{2}+x\left\langle N_{\mathrm{coll}}\right\rangle\right]} .
$$

Now we proceed toward deriving the relationship between the differential cross section per unit pseudorapidity in $p p$ collisions with that in $A+A$ collisions. The distribution of quarks and gluons in a nucleus is different from that in a nucleon with a small effect $(<10 \%)$ of shadowing and European Muon Collaboration (EMC) effects [41]. With a crude approximation one can assume that the gluon distribution in a nucleus is just $A$ times that for a proton, where $A$ is the atomic number. The production is expected to increase by a factor of $A^{2}$ when two nuclei of atomic number $A$ collide in a central way and the pseudorapidity spectrum transforms as [42]

$$
\frac{d \sigma^{A A}}{d \eta}=A^{2}\left(\frac{d \sigma^{p p}}{d \eta}\right) .
$$

Using Eqs. (6) and (7), we write the differential cross section per unit pseudorapidity in terms of charged-particle pseudorapidity distribution for the heavy-ion collisions as

$$
\frac{d \sigma^{A A}}{d \eta}=\frac{A^{2} \sigma_{\mathrm{in}}\left(\frac{d N_{\mathrm{ch}}^{A A}}{d \eta}\right)}{\left[(1-x) \frac{\left\langle N_{\mathrm{part}}\right\rangle}{2}+x\left\langle N_{\mathrm{coll}}\right\rangle\right]} .
$$

A large number of experimental data on the chargedparticle pseudorapidity distribution are available at various center-of-mass energies ranging from RHIC energies like $\sqrt{s_{N N}}=19.6,62.4,130$, and $200 \mathrm{GeV}$ to LHC energies such as $\sqrt{s_{N N}}=2.76$ and $5.02 \mathrm{TeV}[35,43-45]$. In a recent paper by the ALICE experiment [44], the limiting fragmentation phenomenon is studied in the pseudorapidity distribution of charged particles at RHIC and LHC energies. At $\sqrt{s_{N N}}=$ $2.76 \mathrm{TeV}$, the authors have used a double Gaussian function to extrapolate the data in the fragmentation region and find that the phenomenon of LF is observed at this energy.

In Fig. 3, we have shown $d N_{\mathrm{ch}}^{A A} / d \eta /\left(\left\langle N_{\text {part }}\right\rangle / 2\right)$ as a function of $\eta-y_{\text {beam }}$ for various energies from $19.6 \mathrm{GeV}$ to 


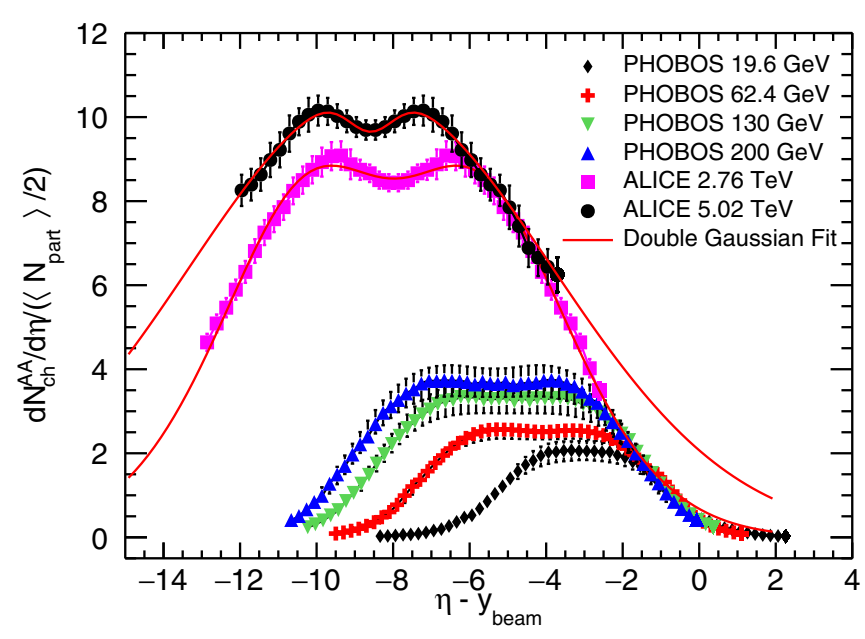

FIG. 3. The number of participant pair normalized pseudorapidity distribution of charged particles $\left(d N_{\mathrm{ch}}^{A A} / d \eta\right)$ in heavy-ion collisions versus $\eta-y_{\text {beam }}$ for various energies. The symbols are experimental data [35,43-45] and the lines are the double Gaussian fits.

5.02 TeV. Due to lack of the experimental data in the fragmentation region at LHC energies, we have used a double Gaussian function to fit and extrapolate the experimental data in the projectile rapidity region. The double Gaussian function used for fitting is given as follows:

$$
f(\eta)=A_{1} e^{\frac{-\eta^{2}}{2 \sigma_{1}^{2}}}-A_{2} e^{\frac{-\eta^{2}}{2 \sigma_{2}^{2}}}
$$

where $A_{1}, A_{2}$ are the amplitudes and $\sigma_{1}, \sigma_{2}$ are widths of the double Gaussian function. This function describes the experimental data very well at LHC energies within uncertainties $[44,45]$. The fitting parameters are given in Table I for $\sqrt{s_{N N}}=2.76$ and $5.02 \mathrm{TeV}$. We observe that the limiting fragmentation phenomenon seems to be violated at $\sqrt{s_{N N}}=$ $5.02 \mathrm{TeV}$, while it is observed at energies from $\sqrt{s_{N N}}=$ $19.6 \mathrm{GeV}$ to $2.76 \mathrm{TeV}$. Despite this, at $\sqrt{s_{N N}}=5.02 \mathrm{TeV}$, the extrapolation of the charged-particle pseudorapidity density scaled with the average number of participants does not show a similar behavior in the fragmentation region as observed at lower energies. The lack of data around the beam rapidity region and the asymmetric values around $\eta=0$ prevent us from drawing any solid conclusion on the behavior observed at the highest LHC energies. It should also be noted here that a Gaussian extrapolation to the fragmentation region is assumption based and its validity is subject to a check against the experimental data.

TABLE I. The values of parameters obtained from the fitting of experimental data of $d N_{\mathrm{ch}} / d \eta$ with the double Gaussian function given by Eq. (9).

\begin{tabular}{lcc}
\hline \hline Parameters & $\sqrt{s_{N N}}=2.76 \mathrm{TeV}$ & $\sqrt{s_{N N}}=5.02 \mathrm{TeV}$ \\
\hline$A_{1}$ & $2592.29 \pm 311.56$ & $2102.16 \pm 28.39$ \\
$A_{2}$ & $959.59 \pm 304.26$ & $1817.56 \pm 37.90$ \\
$\sigma_{1}$ & $3.27 \pm 0.13$ & $4.75 \pm 0.01$ \\
$\sigma_{2}$ & $1.67 \pm 0.23$ & $0.61 \pm 0.14$ \\
\hline \hline
\end{tabular}

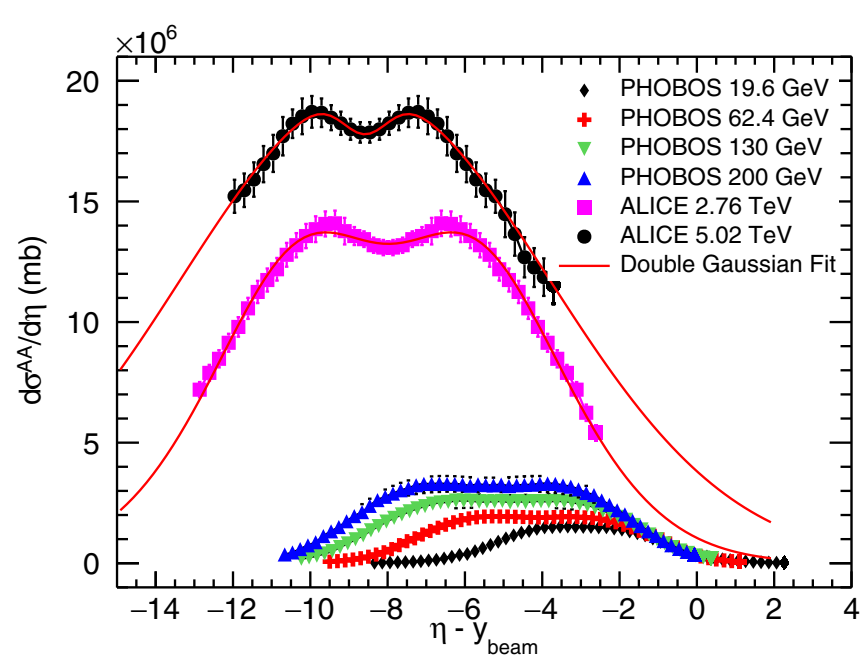

FIG. 4. The differential cross section per unit pseudorapidity $\left(d \sigma^{A A} / d \eta\right)$ as a function of $\eta-y_{\text {beam }}$ for various collision energies. The symbols are experimental points and the lines are double Gaussian fits.

Now we evaluate $d \sigma^{A A} / d \eta$ using Eq. (8) for $\sqrt{s_{N N}}=$ $19.6 \mathrm{GeV}$ to $5.02 \mathrm{TeV}$, taking the $x$ parameters from Ref. [40], which is almost energy independent from RHIC to LHC energies. The inelastic cross sections for various energies are taken from Refs. [34-37]. The Monte Carlo Glauber model [46] is used to calculate the number of participants $\left(N_{\text {part }}\right)$ and the number of binary collisions $\left(N_{\text {coll }}\right)$ at different energies. The differential cross section per unit pseudorapidity for various center-of-mass energies starting from $\sqrt{s_{N N}}=19.6 \mathrm{GeV}$ to $5.02 \mathrm{TeV}$ are shown in Fig. 4 with respect to $\eta-y_{\text {beam }}$. We notice that the limiting fragmentation hypothesis appears to be violated at LHC energies, i.e., at $\sqrt{s_{N N}}=2.76$ and $5.02 \mathrm{TeV}$. These findings suggest that it is very important to consider the energy-dependent $\sigma_{\text {in }}$ to study LF phenomena, particularly at LHC energies.

The experimental data for pseudorapidity distributions of charged particles in the full phase space are not available at the LHC energies. In addition, a double Gaussian extrapolation of $d N_{\mathrm{ch}} / d \eta$ to the $y_{\text {beam }}$ at a given energy seems to introduce an artifact in the spectra, which forbids one to look into the hypothesis of limiting fragmentation. To circumvent this problem, we take the AMPT model in a string melting scenario [47] as shown in Ref. [48] for the most central bins, 0-6\% and $0-5 \%$ for RHIC and LHC energies, respectively. We then compared the measured experimental data for pseudorapidity distribution of charged particles [35,43-45] with the results obtained in the AMPT model. The comparison of experimental data with the AMPT model prediction is shown in Fig. 5. AMPT predictions reproduce the midrapidity and the fragmentation region very well but cannot reproduce experimental data around the peak region $(\eta \sim 0)$ at RHIC energies. For LHC energy at $\sqrt{s_{N N}}=2.76 \mathrm{TeV}$, the AMPT predictions are in good agreement with the experimental data except for the midrapidity region, where the predictions slightly underestimate the measured data. Similarly, for $\sqrt{s_{N N}}=5.02 \mathrm{TeV}$, the predictions from the AMPT model slightly overestimate the 


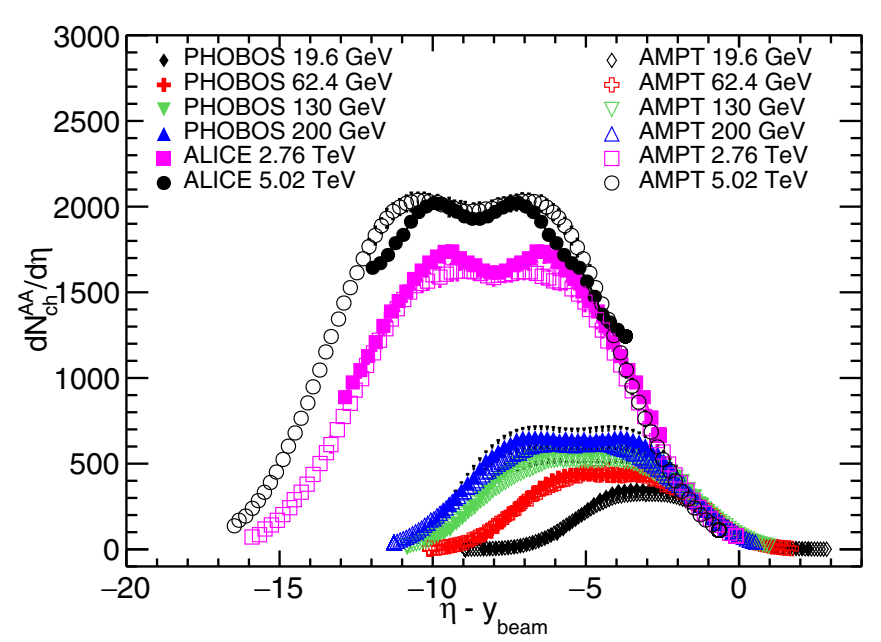

FIG. 5. The comparison of AMPT model predictions with experimental data on $d N_{\mathrm{ch}}^{A A} / d \eta$ versus $\eta-y_{\text {beam }}$ for various energies.

data measured for the $0-5 \%$ centrality bin. In this figure, we see that the phenomenon of longitudinal scaling is observed at RHIC and LHC energies. These findings are also described in Ref. [22], where various transport models like AMPT and the ultrarelativistic quantum molecular dynamics (UrQMD) are used to study this phenomenon. They observed that AMPT (both default and string melting versions) and UrQMD with the default version show longitudinal scaling in pseudorapidity distributions of charged particles at RHIC and LHC energies.

We convert the AMPT results of $d N_{\mathrm{ch}}^{A A} / d \eta$ into $d \sigma^{A A} / d \eta$ using Eq. (8). In Fig. 6, we have shown $d \sigma^{A A} / d \eta$ versus $\eta-y_{\text {beam }}$ to see the longitudinal scaling phenomena in the fragmentation region for different energies from $19.6 \mathrm{GeV}$ to $5.02 \mathrm{TeV}$. Again, we have found a similar observation for the AMPT model as observed in the experimental data, i.e., LF is observed up to RHIC energies in $d \sigma^{A A} / d \eta$ and seems to be violated for LHC energies. Theses findings are very important while discussing the longitudinal scaling hypothesis at LHC energies.

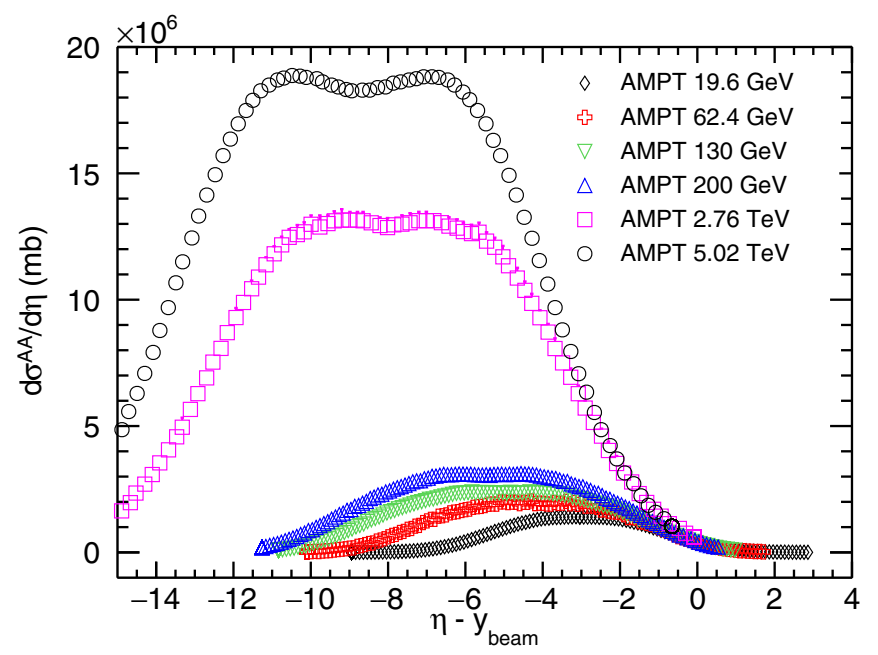

FIG. 6. $d \sigma^{A A} / d \eta$ versus $\eta-y_{\text {beam }}$ using AMPT results.

\section{CONCLUSIONS AND OUTLOOK}

In this work, we have revisited the phenomenon of limiting fragmentation in the pseudorapidity distributions of differential cross sections of charged particles using the energydependent inelastic cross section. The findings of this analysis are as follows:

(i) We have observed the limiting fragmentation phenomenon in the experimental data of $d N_{\mathrm{ch}}^{A A} / d \eta$ from $\sqrt{s_{N N}}=19.6 \mathrm{GeV}$ to $2.76 \mathrm{TeV}$ and it is violated at $\sqrt{s_{N N}}=5.02 \mathrm{TeV}$. Here the double Gaussian function is used to extrapolate the experimental data in the fragmentation region. However, on the basis of the extrapolation method, one cannot infer any exact physics conclusions.

(ii) We have transformed experimental data of $d N_{\mathrm{ch}}^{A A} / d \eta$ to $d \sigma^{A A} / d \eta$ for various energies from $\sqrt{s_{N N}}=$ 19.6 GeV to $5.02 \mathrm{TeV}$ and see the distributions in the rest frame of one of the nuclei. We have found that the LF hypothesis seems to be violated at both the energies, i.e., at $\sqrt{s_{N N}}=2.76$ and $5.02 \mathrm{TeV}$, when one considers the energy-dependent inelastic cross section.

(iii) We have also studied the phenomenon of longitudinal scaling using the AMPT model and employing the same procedure as used for the experimental data. Our studies suggest that AMPT seems to show a possible violation of the limiting fragmentation phenomenon for $d \sigma^{A A} / d \eta$ at LHC energies.

(iv) The hypothesis of LF comes as a natural outcome when particle production follows the Landau hydrodynamics, with a Gaussian pseudorapidity profile.

(v) LF works fine when the hadronic cross section is assumed to be almost independent of energy, which is not the case and hence it is expected to be violated at higher energies. We find that the limiting fragmentation appears to be violated at LHC energies while using the energy-dependent cross section.

(vi) The thermal model with Landau extrapolation to LHC energies for charged particles, predicts a violation of LF [49]. What about photons in this framework? It has been observed that for pions in the thermal model with longitudinal flow, the LF is violated at the LHC energies [50]. What about photons with a longitudinal flow? These need further investigation.

(vii) It is expected that at higher energies, Landau hydrodynamics should fail and we should expect Bjorken boost-invariant hydrodynamics to work out, with the observation of a midrapidity plateau. If LF is a natural outcome of the Landau model, then LF should be violated at LHC for two reasons: (i) failure to see a Gaussian pseudorapidity distribution and (ii) cross sections vary substantially toward higher collision energies.

(viii) At lower collision energies, baryon stopping at the midrapidity is expected and the $d N_{\mathrm{ch}} / d \eta(y)$ is expected to follow a Gaussian-like behavior, which 
could be described by the particle production in the Landau hydrodynamic model. Hence, at these energies, the observation of a limiting fragmentation hypothesis in particle production is expected. But at higher energies, where Landau hydrodynamics fails due to the absence of Gaussian rapidity distribution, $\mathrm{LF}$ is found to be violated.

(ix) Going from the top RHIC energy to the LHC energies, there is an order-of-magnitude increase in the collision energy. Considering at least two units of (pseudo)rapidity overlap for the LF to be valid, the observed $y_{\text {beam }}$ at $\sqrt{s_{N N}}=200 \mathrm{GeV}$ and $5.02 \mathrm{TeV}$ makes hardly any overlap in (pseudo)rapidity. While looking into the possible observation of limiting fragmentation, one looks at spectral overlap in the fragmentation region, which may not be expected as mentioned. Hence, RHIC cannot be combined with LHC while looking for the hypothesis of limiting fragmentation.

(x) Theoretical models are mostly assumption dependent. In order to validate a model, one needs to confront a model to experimental data. We need forward charged particle and photon detectors at the LHC energies to validate the LF hypothesis. In the absence of this, extrapolation of any theoretical findings from midrapidity to extreme forward rapidity would be a speculation sometimes or a mere coincidence, as the physics of particle production is highly rapidity dependent. In view of this, in the present work we have taken the inelastic cross section with the collision energy to study the LF hypothesis. This is the novelty of the present work.

\section{ACKNOWLEDGMENTS}

R.S. acknowledges stimulating discussions with Edward Sarkisyan-Grinbaum. Useful help from Aditya Nath Mishra while preparing the manuscript is highly appreciated. The authors acknowledge the financial support from ALICE Project No. SR/MF/PS-01/2014-IITI(G) of the Department of Science \& Technology, Government of India. This research work used resources of the LHC grid computing center at the Variable Energy Cyclotron Center, Kolkata.
[1] A. Jipa et al., Rom. Rep. Phys. 56, 577 (2004).

[2] W. Kittel and E. A. De Wolf, Soft Multihadron Dynamics (World Scientific, Singapore, 2005).

[3] J. Benecke, T. T. Chou, C. N. Yang, and E. Yen, Phys. Rev. 188, 2159 (1969).

[4] P. Brogueira, J. Dias de Deus, and C. Pajares, Phys. Rev. C 75, 054908 (2007).

[5] F. Gelis, A. M. Stasto, and R. Venugopalan, Eur. Phys. J. C 48, 489 (2006).

[6] J. Jalilian-Marian, Phys. Rev. C 70, 027902 (2004).

[7] Y. P. Nikitin and I. L. Rozental, Theory of Multiparticle Production Processes (Harwood Academic, Reading, PA, 1988).

[8] G. Wolschin, J. Phys. G 40, 045104 (2013).

[9] G. Wolschin, Eur. Phys. J. A 5, 85 (1999).

[10] B. B. Back et al. (PHOBOS Collaboration), Phys. Rev. C 74, 021902(R) (2006).

[11] G. Alner et al. (UA5 Collaboration), Z. Phys. C 33, 1 (1986).

[12] B. Alver et al. (PHOBOS Collaboration), Phys. Rev. Lett. 102, 142301 (2009).

[13] J. Adams et al. (STAR Collaboration), Phys. Rev. Lett. 95, 062301 (2005).

[14] J. Adams et al. (STAR Collaboration), Phys. Rev. C 73, 034906 (2006).

[15] B. I. Abelev et al. (STAR Collaboration), Nucl. Phys. A 832, 134 (2010).

[16] B. B. Back et al. (PHOBOS Collaboration), Phys. Rev. C 72, 031901(R) (2005).

[17] B. B. Back et al. (PHOBOS Collaboration), Phys. Rev. Lett. 91, 052303 (2003).

[18] B. Abelev et al. (ALICE Collaboration), Phys. Rev. Lett. 110, 032301 (2013)

[19] B. B. Abelev et al. (ALICE Collaboration), Eur. Phys. J. C 75, 146 (2015).
[20] A. Bialas and M. Jezabek, Phys. Lett. B 590, 233 (2004).

[21] J. Ruan and W. Zhu, Phys. Rev. C 81, 055210 (2010).

[22] M. Nasim, C. Jena, L. Kumar, P. K. Netrakanti, and B. Mohanty, Phys. Rev. C 83, 054902 (2011).

[23] J. Bleibel, L. V. Bravina, and E. E. Zabrodin, Phys. Rev. D 93, 114012 (2016).

[24] A. Stasto, Nucl. Phys. A 854, 64 (2011).

[25] G. Torrieri, Phys. Rev. C 82, 054906 (2010).

[26] E. K. Sarkisyan-Grinbaum, A. N. Mishra, R. Sahoo, and A. S. Sakharov, arXiv:1803.01428.

[27] E. K. G. Sarkisyan, A. N. Mishra, R. Sahoo, and A. S. Sakharov, Phys. Rev. D 93, 054046 (2016); 93, 079904(E) (2016).

[28] C. Loizides, J. Kamin, and D. d'Enterria, Phys. Rev. C 97, 054910 (2018).

[29] L. D. Landau, Izv. Akad. Nauk Ser. Fiz. 17, 51 (1953).

[30] P. Carruthers, Ann. N. Y. Acad. Sci. 229, 91 (1974).

[31] P. Steinberg, Acta Phys. Hung. A 24, 51 (2005).

[32] J. Steinheimer and M. Bleicher, Eur. Phys. J. A 48, 100 (2012).

[33] J. D. Bjorken, Phys. Rev. D 27, 140 (1983).

[34] G. Aad et al. (ATLAS Collaboration), Nat. Commun. 2, 463 (2011)

[35] B. B. Back et al., Nucl. Phys. A 757, 28 (2005).

[36] B. Abelev et al. (ALICE Collaboration), Phys. Rev. C 88, 044909 (2013).

[37] J. Adam et al. (ALICE Collaboration), Phys. Rev. Lett. 116, 222302 (2016).

[38] P. Carruthers and M. Doung-van, Phys. Rev. D 8, 859 (1973).

[39] X. N. Wang and M. Gyulassy, Phys. Rev. Lett. 86, 3496 (2001).

[40] A. Nath Mishra, R. Sahoo, P. Sahoo, P. Pareek, N. K. Behera, and B. K. Nandi, Eur. Phys. J. A 52, 319 (2016).

[41] L. Frankfurt, M. Strikman, and S. Liuti, Nucl. Phys. A 527, 571C (1991).

[42] R. C. Hwa, in Proceedings of Relativistic heavy-ion collisions, Beijing (Oregon University, Eugene, 1989), pp. 239-309. 
[43] B. B. Back et al. (PHOBOS Collaboration), Phys. Rev. C 74, 021901 (2006).

[44] E. Abbas et al. (ALICE Collaboration), Phys. Lett. B 726, 610 (2013).

[45] J. Adam et al. (ALICE Collaboration), Phys. Lett. B 772, 567 (2017).

[46] M. L. Miller, K. Reygers, S. J. Sanders, and P. Steinberg, Ann. Rev. Nucl. Part. Sci. 57, 205 (2007).
[47] Z. W. Lin, C. M. Ko, B. A. Li, B. Zhang, and S. Pal, Phys. Rev. C 72, 064901 (2005).

[48] S. Basu, T. K. Nayak, and K. Datta, Phys. Rev. C 93, 064902 (2016).

[49] J. Cleymans, J. Strumpfer, and L. Turko, Phys. Rev. C 78, 017901 (2008).

[50] S. K. Tiwari and R. Sahoo, Eur. Phys. J. A 52, 365 (2016). 